\title{
PITIRIASIS VERSICOLOR POR Malassezia pachydermatis: CASO CLÍNICO
}

\section{(Pityriasis versicolor caused by Malassezia pachydermatis: Clinical case)}

\author{
Cruz Ch, Rodrigo*. Vieille O, Peggy*., \\ Giusiano Gustavo**, Sosa Maria de los Ángeles** \\ *Cátedra de Micologia, Escuela de Medicina, Universidad de Valparaíso. \\ Casilla 92 V Valparaíso, Chile. \\ ** Departamento de Micología, Instituto de Medicina Regional, \\ Universidad nacional del Nordeste, Resistencia, Chaco. Argentina.
}

Palabras clave: Pitiriasis versicolor, Malassezia pachydermatis, Chile. Key words: Pityriasis versicolor, Malassezia pachydermatis, Chile.

\section{RESUMEN}

Se presenta un caso clínico de pitiriasis versicolor por M. pachydermatis en una paciente de 11 años de edad, diagnosticado mediante clínica, morfofisiología y biología molecular. Malassezia pachydermatis es parte de la microbiota común de diversos mamíferos domésticos y animales salvajes. En éstos se reconoce como agente causal de dermatitis y otitis externa. En humanos se han descrito escasas infecciones ya sea superficiales como invasoras, lo que puede atribuirse a la dificil transmisión y adaptación a partir de los animales domésticos. Se comentan los aspectos biológicos y epidemiológicos de esta infección zoonótica.

\section{INTRODUCCIÓN}

La pitiriasis versicolor corresponde a una micosis superficial crónica y benigna causada por levaduras del género Malassezia. Clínicamente se caracteriza por máculas de diferentes tamaños, finamente descamativas, hipo y/o hiperpigmentadas y localizadas preferentemente en cuello y tronco (Vilata, 2006; Tango \& Vargas, 2009).

La descripción de esta enfermedad data desde el siglo XIX cuando Eichstedt, en 1846 observó la presencia de levaduras y filamentos en la piel de pacientes con lesiones hipopigmentadas, denominación que le fue otorgada posteriormente por Sluyter (1847), quien la describió en su tesis doctoral como una enfermedad causada por una planta parásita (Ochoa de Quinzada, 2006). En 1889, Baillon propone la creación del género Malassezia (con M. furfur como especie tipo), en honor al histólogo

Recibido el 12 de Noviembre 2010

Aceptado el 29 de Diciembre 2010

\section{ABSTRACT}

A clinical case of pityriasis versicolor caused by M. pachydermatis in an 11 year old patient which was diagnosed by means of clinic, morphophysiology and molecular biology is presented. Malassezia pachydermatis is part of regular microbiota in several domestic mammals as well as wild animals. It is recognized as the causing agent of dermatitis and external otitis in the latter. As to human beings, superficial infections have rarely been given an invasive character what can be explained by the difficult transmission and adaptation coming from domestic animals. Biological and epidemiological aspects of this zoonotic infection are described.

Malassez, quien en 1874 informó presencia de células brotantes de varias formas en el estrato córneo de pacientes con diversas enfermedades de la piel. Años más tarde, Sabouraud en 1904, creó el género Pityrosporum para la fase levaduriforme del hongo (Vilata 2006; Ochoa de Quinzada, 2006; Giusiano, 2006). A partir de este momento y hasta 1986, año en que se aceptó su sinonimia, hubo coexistencia de ambos géneros: Malassezia y Pityrosporum. Por prioridad cronológica el nombre quedó establecido como Malassezia.

La especie Pityrosporum pachydermatis fue introducida por Weidman en 1925 para denominar a una especie no lipofílica aislada desde animales. Una década después, Dodge propone incluirla dentro del género Malassezia (Gordon, 1979). Varios años más tarde, Simmons \& Guého (1990), describen otra especie (M. sympodialis).

En 1996, Guého, Midgley \& Guillot revisan la taxonomía del género Malassezia a través de morfología, fi- 
siología, ultraestructura y biología molecular, logrando identificar 4 nuevas especies: M. globosa, M. restricta, M. obtusa y M. slooffiae. Actualmente, están incorporadas al género $M$. dermatis, $M$. nana, M. japonica, $M$. yamatoensis, M. equina y M. caprae (Hirai, et al, 2004; Sugita, et al., 2002, 2003, 2004). Actualmente una nueva especie $M$. equi, ha sido descrita pero no completamente caracterizada. (Ashbee, 2007).

Las levaduras del género Malassezia pertenecen a la división Basidiomycota, son ovales o elipsoidales, de tamaño pequeño, entre 2 y $7 \mu \mathrm{m}$, de reproducción enteroblástica con brotación monopolar repetitiva, que origina en la célula madre una cicatriz en forma de collar (Guillot, et al., 1996). Del total de las especies, M.pachydermatis se diferencia fisiológicamente de las restantes al no presentar lipodependencia (Chryssanthou, 2001; Villata, 2006), también es la única especie considerada zoofílica que se encuentra en piel y canal auditivo externo de perros, gatos y otros mamíferos, asociándose a otitis externa y dermatitis seborreica canina. Sin embargo, raramente en humanos se ha descrito como agente causal de pitiriasis versicolor, en dermatitis seborreica y en fungemia nosocomial en recién nacidos prematuros y adultos inmunocomprometidos (Guerra, 2004; Yi-Ming, et al., 2006; Chryssanthou, et al ., 2001; Chang et al., 1998).

La literatura nacional empieza a integrarse en los estudios morfofisiológicos y moleculares de los integrantes del género, causantes de micosis cutáneas tanto en humanos como en patología veterinaria (Rendic et al., 2003; Candido, et al., 1996; Sotomayor, 2005).

Aparentemente, no se ha publicado en Chile ningún aislamiento de M.pachydermatis en humanos asociada a pitiriasis versicolor ni a otros cuadros clínicos, probablemente debido a que los informes se basan aún principalmente en los exámenes micológicos directos sin los cultivos correspondientes.

Comunicamos el caso de una paciente de 11 años de edad con residencia en la ciudad de Valparaíso, quien presentaba lesiones hipopigmentadas en tórax, características de pitiriasis versicolor. Se identificó como agente causal a $\boldsymbol{M}$.pachydermatis.

\section{CASO CLÍNICO}

Paciente de 11 años de edad, de sexo femenino y nacionalidad francesa, residente en Chile desde hace 2 años, sin antecedentes mórbidos. Dueña de un gato, al cual mantiene en brazos frecuentemente. Consulta al dermatólogo por presentar lesiones hipopigmentadas en cuello y región anterior del tórax, sin prurito, sin dolor ni signos inflamatorios, de aproximadamente 3 meses de evolución (Figura 1A), por lo que se le solicitó examen directo y cultivo para hongos.

\section{Estudio micológico.}

El examen directo se realizó con cinta adhesiva y tinción con safranina, observándose levaduras ovoideas de 2-2,5 x 3-6 $\mu \mathrm{m}$ con base de brotación ancha (Figura 1B) de morfología no similar a M. furfur. Debido a esto, a partir de un raspado de piel se cultivaron las escamas en agar oliva adicionado con Tween 80 y en agar Sabouraud a $32^{\circ} \mathrm{C}$ por 7 días, lográndose desarrollo de colonias blancas- amarillentas, cremosas y frágiles, con mayor crecimiento en el primero ( hasta $10 \mathrm{~mm}$ ) y hasta $3 \mathrm{~mm}$ en el segundo, además de pruebas complementarias como catalasa $(+)$, ureasa $(+)$, esculina $(+)$ y crecimiento a $37 \mathrm{y}$ $40^{\circ} \mathrm{C}$. Con estos resultados, la cepa fue identificada como M.pachydermatis.

\section{Estudio molecular}

Con la finalidad de ratificar su tipificación mediante la técnica PCR-RFLP, la cepa fue enviada a la cátedra de Micología de la Universidad del Nordeste de Argentina. La extracción de ADN de las levaduras aisladas se llevó a cabo por shock térmico. El ADN obtenido se amplificó utilizando los cebadores descritos por Mirhendi et al., (2004). (5'-TAA CAA GGATTC CCC TAG TA-3' y 5'-ATT ACG CCA GCA TCC TAA G -3').

Los productos de la PCR fueron sujetos a RFLP utilizando las enzimas de restricción Cfo I (Promega) y Mbo I (Fermentas). Los fragmentos digeridos fueron separados por electroforesis en gel de agarosa al 2,5\% a $96 \mathrm{~V}$ durante 90 minutos y teñidos con bromuro de etidio $(0.5 \mu \mathrm{g} / \mathrm{ml})$. El estudio fue compatible con M. pachydermatis concordando de esta forma con las determinaciones morfofisiológicas. (Figura 2)

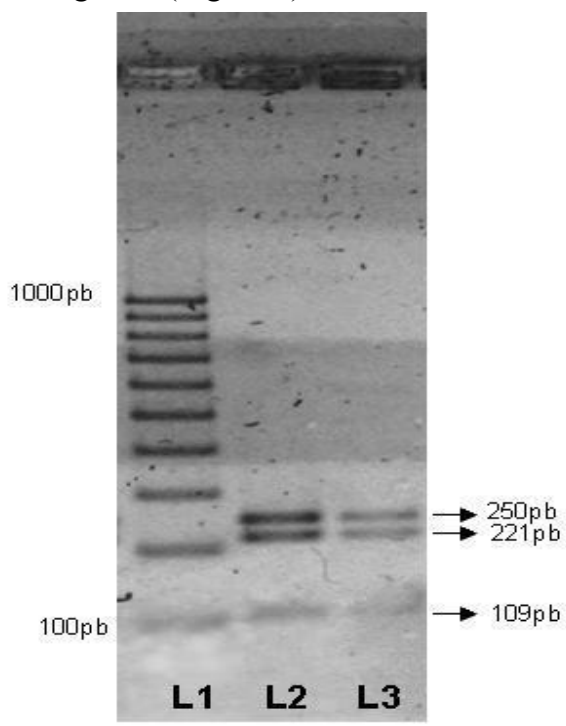

Figura 2. PCR-RFLP (Cfo I), L1: MPM L2: CBS 1879 M. pachydermatis, L3: Cepa X 


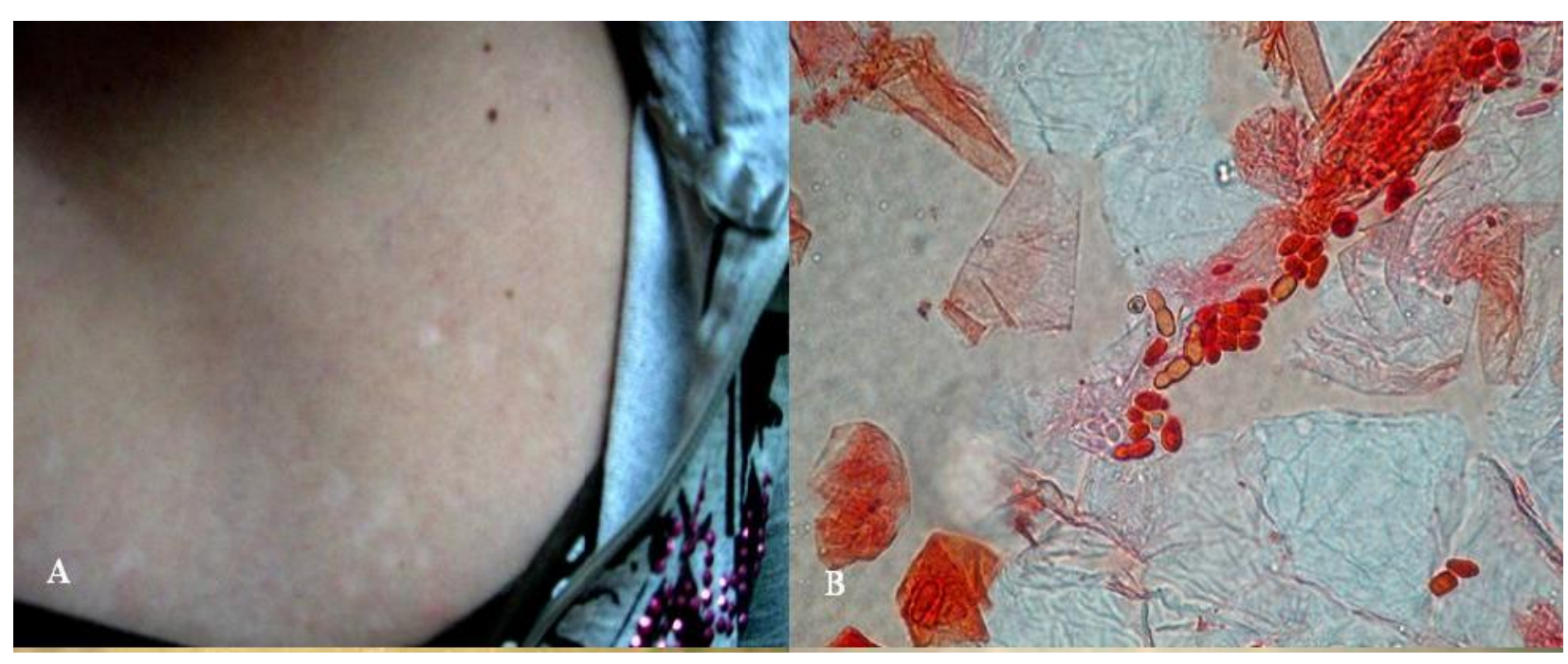

Figura 1: A. Lesiones hipopigmentadas en tórax B. Tinción con safranina de M. pachydermatis en muestra tomada con cinta adhesiva

\section{DISCUSIÓN}

M. pachydermatis, es parte de la microbiota de diversos mamíferos domésticos (perros, gatos y equinos) y animales salvajes (primates, rinocerontes, aves acuáticas, pájaros y carnívoros) (Rejas, 2008; Volk et al., 2010; Guillot,1988). Se la reconoce como agente causal de diversas patologías que afectan a los animales, aislándose con frecuencia en casos de dermatitis y otitis externa (Guillot \& Bond, 1999; Raabe et al., 1998; Pier et al., 2000; Huan et al., 1998; Gaitanis, 2002, Sotomayor, 2005).

La literatura presenta escasas publicaciones que relacionen a $\boldsymbol{M}$. pachydermatis con patologías humanas. Bandhaya (1993), en Tailandia, estudió la colonización de M. furfur y M. pachydermatis en 200 voluntarios sanos entre 20 y 30 años de edad, en 10 sitios diferentes del cuerpo. En todos los sujetos estudiados se aisló M. furfur, con recuen-tos altos en la región de la cabeza y el tronco superior, en cambio, solo en el $12 \%$ de los individuos se aisló M. pachydermatis en recuentos bajos en palmas y cabeza.

Prohic \& Kasumagic-Halilovic (2009), en BosniaHerzegovina, identificaron de entre 160 muestras de piel de pacientes con pitiriasis versicolor, dermatitis seborreica, psoriasis y piel sana, solo 1 caso de $\boldsymbol{M}$. pachydermatis, la que se relacionó a dermatitis seborreica; concluyendo que esta especie no es parte de la micota normal humana, sino una infección zoonótica.

En Argentina, Giusiano et al. (2010), reporta el primer caso de $\boldsymbol{M}$. pachydermatis aislada desde lesiones con pitiriasis versicolor en su región (Resistencia, nordeste de Argentina). En Chile, Rendic et al. (2003), estudiaron a 160 pacientes, encontrando presencia de Malassezia spp. en $76,5 \%$ de los pacientes del grupo con dermatitis sebor- reica y en $82 \%$ del grupo control (sin diferencia significativa). Se logró identificar a nivel de especie 50 levaduras, cuya presencia fue la siguiente: 36 M. globosa (72\%), 7 M. sympodialis (14\%), 5 M. furfur (10\%) y 2 M. slooffiae (4\%). No obtuvieron aislamiento para M. pachydermatis.

En Estados Unidos, Larocco et al. (1988), estudiaron retrospectivamente los cultivos obtenidos de 507 niños hospitalizados en la unidad de cuidados intensivos neonatal entre octubre 1985 y enero 1987. En ocho pacientes fue aislada $M$. pachydermatis en una o más muestras clínicas: sangre (4 pacientes), puntas de CVC (3), orina (4), LCR (1), secreción ocular (1), secreción del oído (1) y aspirado traqueal (1). Todos los niños fueron prematuros y presentaban múltiples complicaciones. Siete niños sintomáticos recibieron múltiples antibióticos, además de hiperalimentación parenteral (incluidos lípidos) por CVC. Mickelson et al. (1988), en el mismo año, reportaron tres lactantes con sepsis por M. pachydermatis en UCI neonatal. Todos los niños tenían al nacer pesos menores a 1.000 gramos, además de tener CVC con hiperalimentación lípidica. Chang et al. (1998), describieron 15 pacientes con cultivos positivos para M. pachydermatis en la unidad de cuidados intensivos neonatal; 8 en infecciones del torrente sanguíneo, 2 en infecciones de vías urinaria, 1 en meningitis y 4 colonizaciones asintomáticas ( 2 en aspirado traqueal, 1 en piel y 1 en punta de catéter). En ese trabajo recolectaron muestras de cara, cuello, ingle, espalda, ano y de los sitios de cateterismo intravascular en 23 niños internados en la sala de cuidados intensivos. También se obtuvieron muestras de manos de nueve trabajadores de la salud presentes en la sala en el cambio de turno y muestras de las orejas de las mascotas de los mismos. Una enfermera y el 31\% de las mascotas estudiadas se encontraban colonizadas por $\boldsymbol{M}$. 
pachydermatis. Concluyeron que uno o más trabajadores de la salud fueron los portadores para la introducción de esta levadura en la sala de cuidados intensivos por una transferencia desde las mascotas a sus amos.

Chryssanthou et al. (2001), reportaron el aislamiento de este agente en una unidad de cuidados intensivos neonatal de Suecia; 6 en hemocultivos y 2 en secreción traqueal. El uso prolongado de catéteres centrales y las formulaciones parenterales de lípidos fueron factores de riesgo para estas infecciones.

A pesar que morfológicamente $M$. pachydermatis presenta ciertas diferencias que permiten su diagnóstico, la mayoría de los exámenes clínicos micológicos en pitiriasis versicolor informan solo la presencia positiva de una levadura gemante con o sin micelio corto asociado, agrupadas de variadas formas entre las células superficiales de la epidermis, clasificándolas como Malassezia complex, o simplemente como Malassezia spp. Esta situación se asocia principalmente a las dificultades que implica la identificación, debido a que en general, el tratamiento no varía mayormente. Esta situación, que excluye la determinación correcta del agente causante a nivel de especie, ha impedido prácticamente nuevos aportes a los datos epidemiológicos del país.

En Chile, no se han reportado casos de infecciones cutáneas o de fungemias por M. pachydermatis en humanos. Este raro caso de pitiriasis versicolor constituye un incentivo para seguir estudiando la diversidad de sus agentes etiológicos mediante los cultivos adecuados que permitan no solo aportes taxonómicos, sino mejores conocimientos de sus roles en la invasividad y patogenia de estas levaduras

\section{REFERENCIAS}

Ashbee, H.R. (2007). Update on genus Malassezia. MedMycol. $45: 287-303$

Aspíroz, M.; Moreno, L. \& Rubio, M. (1997). Taxonomía de Malassezia furfur: estado de la cuestión . Rev. Iberoam. Mico. 14:147-149

Bandhaya, M. (1993). The distribution of Malassezia furfur and Malassezia pachydermatis on normal human skin. Southeast Asian J Trop Med Public Health. Jun;24:343-346

Cabañes, J.; Theelen, B.; Castellá, G. \& Boekhout, T. (2007). Two newlipid-dependent Malassezia species from domestic animals. FEMS Yeast Res 7:1064-1076

Candido, R..; Zaror, L.; Fisheman, O.; Gregorio, Z.; Isidoro, T.; Castanha, L. (1996). Actividad de antisépticos en Malassezia pachydermatis aislada de oído externo en perros y gatos. Boletín Micológico. 11:51-54

Chang, H.; Miller, H.; Watkins, N.; Arduino, M.; Ashford, D.; Midgley, G.; Aguero,S.; Pinto-Powell, R.; Fordham von
Reyn, C.; Edwards, W.; McNeil, M.; Jarvis, W.; Pruitt. R. (1998). An Epidemic of Malassezia pachydermatis in an Intensive Care Nursery Associated with Colonization of Health Care Workers' Pet Dogs. N. Engl. J. Med. 338:706-711

Chryssanthou, E.; Broberger, U. \& Petrini, B. (2001). Malassezia pachydermatis fungaemia in a neonatal intensive care unit. Acta Pediatr. 90:323-327

Gaitanis, G.; Velegraki, A.; Frangoulis, E.; Mitroussia, A.; Tsigonia, A.; Tzimogianni, A.; Katsambas, A.; Legakis, N. (2002). Identification of Malassezia species from patient skin scales by PCR-RFLP Clin. Microbiol. Infect.; 8: 162-173

Giusiano, G. (2006). Malassezia Estado del conocimiento y perspectivas en su estudio Revista Argentina de Microbiología 38: $41-48$

Giusiano, G.; Sosa, M.; Rojas; F.; Toma Vanacore, S. \& Mangiaterra, M. (2010). Prevalence of Malassezia species in pityriasis versicolor lesions in northeast Argentina. Rev. Iberoam. Micol. 27: 71-74

Gordon, MA. (1979). Malassezia, Pityrosporum pachydermatis (Weidman) Dodge 1935. Sabouraudia; 17:305-309

Guého, E.; Midgley, G. \& Guillot, J. (1996). The genus Malassezia with description of four new species. Antonie van Leeuwenhoek; 69:337-355

Guerra, T. \& Corrales, R. (2004). Incidencia de Malassezia pachydermatis en procesos dermatológicos caninos. Revista electrónica de Veterinaria 12:1695-7504

Guillot, J. \& Bond, R. (1999). Malassezia pachydermatis: a review. Med. Mycol. 37:295-306

Guillot, J.; Guého, E.; Lesourd, M.; Midgley, G.; Chévrier, G.; Dupont, B. (1996). Identification of Malassezia species. A practical approach. J. Mycol. Med. 6: 103-110

Guillot, J.; Guého, E.; Mialot, M. \& Chermette, R. (1998) Importance des levures du genre Malassezia en dermatologie vétérinaire. Le Point Veterinaire, 29: 691-701.

Hernández-Molina, JM. (1993) Revisión bibliográfica sobre Malassezia (Pityrosporum): taxonomía y su importancia en las infecciones sistémicas. Rev. Iberoam. Micol. 10:24-28

Hirai, A.; Kano, R.; Makimura, K.; Duarte, ER.; Hamdan, JS.; Lachance, MA.; Yamaguchi, H.; Hasegawa, A. (2004) Malassezia nana sp. nov., a novel lipid-dependent yeast species isolated from animals. Int. J. Syst. Evol. Microbiol. 54:623-627

Larocco, M.; Dorenbaum, A.; Robinson, A. \& Pickering, LK. (1988). Recovery of Malassezia pachydermatis from eight infants in a neonatal intensive care nursery: clinical and laboratory features. Pediatr. Infect. Dis. J. 7:398-401

Ochoa de Quinzada, M. (2006). Estudio de las especies de Malassezia relacionadas con la patología cutánea, pitiriasis versicolor en Panamá. Tesis doctoral. Universidad de Panamá, Panamá.

Mickelson, P.A.; Viano-Paulson, M.C.; Stevens, D.A. \& Diaz, P.S. (1988). Clinical and microbiological features of infection with Malassezia pachydermatis in highrisk infants. J. Infect. Dis. 157:1163-1168 
Mirhendi, H.; Makimura, K.; Zomorodian, K.; Yamada, T.; Sugita, T.; Yamaguchi, H. (2005). A simple PCR-RFLP method for identification and differentiation of 11 Malassezia species. J. Microbiol. Methods 61:281-284

Pier, A.C.; Cabañes, F.J.; Chermette, R.; Ferreiro, L.; Guillot, J.; Jensen, H.E. \& Santuario, J.M. (2000). Prominent animal mycoses from varius regions of the world. Med. Mycol. 38: $47-58$

Prohic, A.; \& Kasumagic-Halilovic, E. (2009). Identification of Malassezia pachydermatis from healthy and diseased human skin. Med Arh. 63: 317-319

Raabe, P.; Mayser, P. \& Weib, R. (1998). Demonstration of Malassezia furfur and $\boldsymbol{M}$. sympodialis together with $\boldsymbol{M}$. pachydermatis in veterinary specimens mycoses. Mycoses 41:493500

Rejas, J. (2008). Dermatitis canina por Malassezia - Canine Malassezia dermatitis . Revista electrónica de Veterinaria 12:16957504

Rendic, E. Díaz, C. \& Fich, F. (2003). Caracterización de especies del género Malassezia en pacientes con dermatitis seborreica y en controles. Rev. Med. Chil. 131:1295-1300

Simmons, RB. \& Guého, E. (1990). A new species of Malassezia Mycol. Res. 1146-1149

Sotomayor, C. (2005). Características epidemiológicas descriptivas y factores de riesgo en otitis canina en pacientes atendidos en el hospital veterinario de la Universidad Austral de Chile durante el periodo 1998-2003. Memoria de titulo. Valdivia, Chile.
Sugita, T.; Takashima, M.; Kodama, M.; Tsuboi, R.; Nishikawa, A. (2003). Description of a new yeast species, Malassezia japonica, and its detection in patients with atopic dermatitis and healthy subjects.J.Clin.Microbiol.41:4695-4699

Sugita, T.; Takashima, M.; Shinoda, T.; Suto, H.; Unno, T.; Tsuboi, R. Ogawa, H.; Nishikawa, A. (2002). New yeast species, Malassezia dermatis, isolated from patients with a topic dermatitis. J. Clin. Microbiol.40:1363-1367

Sugita, T.; Tajima, M.; Takashima, M.; Amaya, M.; Saito, M.; Tsuboi, R.; Nishikawa, A. (2004). A new yeast, Malassezia yamatoensis, isolated from patient with seborrhoeic dermatitis, and its distribution in patients and healthy subjects. Microbiol. Immunol.48:576-583

Tango, E. \& Vargas, J. (2009). Caracterización fenotípica de las especies del género Malassezia aisladas de pacientes con pitiriasis versicolor en Santa Cruz Bolivia. Rev. Enfermedades Infecciosas y Tropicales 1:33-36

Vilata, J.J. (2006). Micosis cutáneas. Editorial Médica Panamericana

Volk, AV. ; Belyavin, CE. ; Varjonen, K. ; Cadiergues, MC. ; Stevens, KB. ; Bond, R. (2010). Malassezia pachydermatis and M. nana predominate amongst the cutaneous mycobiota of Sphynx cats. J Feline Med Surg. 12:917-922

Yi-Ming, F. ; Wen-Ming, H.; Shun-Fan, L.; Guo-Feng, W. ; Kuan, L, ; M.; Rong-Yi, C. (2006). Granulomatous Skin Infection Caused by Malassezia pachydermatis in a Dog Owner. Arch. Dermatol.142:1181-1184 